\title{
Preoperative Diagnosis of Hepatic Sinus Obstruction Syndrome Caused by Tusanqi and Evaluation of Therapeutic Effects after TIPS by Imaging and Pathology
}

Yusheng Heף, Kaicai Liuđ, Weifu Lv, Chunze Zhou, Delei Cheng, Yijiang Zhu, Dong Lu, Zhengfeng Zhang, Jingkun Xiao, Xingming Zhang,

Department of interventional radiology, Anhui provincial hospital, The First Affiliated Hospital of USTC,Division of Life Sciences and Medicine,University of Science and Technology of China, hefei, anhui, china

"These authors contributed equally to this work.

\section{${ }^{*}$ Corresponding author}

Weifu Lv

Department of interventional radiology, Anhui provincial hospital, The First Affiliated Hospital of USTC,Division of Life Sciences and Medicine,University of Science and Technology of China

E-mail:ahsllkc@163.com

\begin{abstract}
BACKGROUND

Transjugular intrahepatic portosystemic shunt(TIPS) is a method for the treatment of hepatic sinus obstruction syndrome (HSOS). This study investigated the preoperative diagnosis and prognosis of hsos caused by Tushanqi from the perspective of imaging and pathology.
\end{abstract}

\section{METHOD}

TIPS was administered to our hospital for 19 patients with HSOS who had a history of taking Tusanqi soaking liquor or Chinese medicine between September 2014 to December 2018. They underwent enhanced abdominal CT or MR scans on the day before surgery and one month after surgery. ultrasound-guided liver biopsy, and specimens for HE staining. 


\section{RESULTS}

Preoperative imaging showed increased liver volume, uneven density, moderate to severe effusion in the abdominal cavity, thickening and distortion of the hepatic artery, uneven perfusion of the liver parenchyma, slender or unclear three hepatic veins, and slender inferior vena cava or flat, but still unobstructed. Pathological examination showed extensive edema and degeneration of hepatocytes. hepatic sinus was obviously congested, dilated, fibrous connective tissue proliferated, and expanded venules in the interfiber space.One month after operation: the liver volume was smaller than before, no obvious abnormalities were found in the liver parenchyma, and the peritoneal effusion was significantly less or even disappeared.Postoperative pathology showed marked improvement of hepatocellular edema, slight dilatation and congestion of hepatic sinus.

\section{CONCLUSIONS}

Imaging and pathology can help the diagnosis of HSOS by Tusanqi , but also evaluate the therapeutic effect of TIPS on the disease.

Key words: tusanqi; hepatic sinus obstruction syndrome; CT; MRI; pathology

\section{Background}

Hepatic sinus obstruction syndrome (HSOS), also known as hepatic venule occlusion syndrome, refers to a series of clinical symptoms caused by non-thrombotic stenosis and occlusion of the central and inferior lobular veins of the hepatic lobule, leading to retrosinular portal hypertension, which is a rare but life-threatening clinical syndrome ${ }^{[1-2]}$. The most important and common cause of HSOS in Western countries is the use of high-dose chemotherapy in recipients of haematopoietic stem cell transplantation, although many other factors, including alcohol use, the use of oral contraceptives and radiation damage, are related to the pathogenesis of HSOS ${ }^{[3]}$. In China, most HSOS patients are treated with plants containing pyrrolidine alkaloids. Some traditional herbs, including Tusanqi, senecio, and cowpea, can be pathogenic. 
The diagnostic criteria used for HSOS were mostly based on the Seattle criterion ${ }^{[4]}$ and Baltimore criterion ${ }^{[5]}$, which have an accuracy of $89 \%$ and $95 \%$, respectively ${ }^{[6]}$. The main cause of HSOS in China is taking plants containing pyrrolidine alkaloids (PAs, also pyrrolizidineal alkoids). The domestic reported proportion of HSOS caused by taking Tusanqi is $50.0 \%-88.6 \%{ }^{[7]}$. Therefore, this standard is not applicable to China. The Nanjing Standard for HSOS Diagnosis in China was formulated in 2017 ${ }^{[8]}$.

At present, there is no unified treatment for HSOS at home or abroad. Diuresis, liver protection, albumin supplementation and other symptomatic supportive treatments are the basic measures used in these patients. Among HSOS patients who receive only basic treatment, most studies reported in the literature ${ }^{[9-10]}$ show that their mortality rate is over $40 \%$. There is no specific drug treatment for HSOS. Studies have shown that heparin anticoagulation can be used early in these patients $\left.{ }^{[11}\right]$. Prostaglandins and Salvia miltiorrhiza can improve microcirculation to a certain extent but lack support from large sample data. Foreign reports show that ${ }^{[12]}$ defibrotid (DF) is effective, but it is not on the market in China. The efficacy of PAs in HSOS is uncertain. Glucocorticoids are considered effective because of their anti-inflammatory effects. A small sample of case reports ${ }^{[13]}$ show that the use of glucocorticoids can improve the cure rate of HSOS, but no randomized controlled study has tested this, so the use of glucocorticoids remains controversial. Transjugular Intrahepatic Portosystemic Shunt (TIPS) can reduce ascites and portal hypertension. TIPS was not recommended for HSOS caused by HSCT in the Italian TIPS Management Consensus of 2016 ${ }^{[14]}$. However, in 2017, China's consensus on the diagnosis and treatment of HSOS caused by PAs was included. It has been suggested that TIPS can be used to control refractory ascites and reduce portal hypertension in patients with poor therapeutic effects $^{[8]}$. This study improves the diagnosis of HSOS by employing imaging and pathology and retrospectively analysed the efficacy of TIPS in the treatment of HSOS; these results provide a certain clinical basis for clinical diagnosis and treatment.

\section{2. methods}




\subsection{Study population}

Nineteen patients with HSOS complicated with refractory ascites caused by mistaken ingestion of Tusanqi from June 2016 to June 2018 were selected for TIPS treatment. Fourteen male and 5 female patients were included. The ages ranged from 30 to 78 years. All patients met the following requirements: (1) a clear history of taking PA

plants; (2) abdominal distention and pain in the liver region with ascites; (3) liver injury; (4) typical CT or MR manifestations; (5) liver biopsy showed the expansion of sinus congestion; and (6) ascites that did not subside after conservative medical treatment. The exclusion criteria were as follows: (1) patients with viral, autoimmune, metabolic, hereditary and other liver diseases and (2) poor coagulation mechanism or contraindication for liver puncture.

\subsection{Transjugular Intrahepatic Portosystemic Shunt}

The patient took the supine position, and the right jugular skin was routinely disinfected; the clinician wore sterile gloves, laid sterile hole towels on the site, and successfully punctured the internal jugular vein with the Seldinger technique. After that, under guidance by a $5 \mathrm{~F}$ multi-functional catheter, the guide wire was sent to the right hepatic vein for the right hepatic venous shadow. After successful puncture of the femoral artery with $2 \%$ lidocaine local anaesthesia, a $5 \mathrm{~F}$ sheath was inserted along the guide wire, and a 5F hepatic duct was inserted with the help of the guide wire. The aortic arch was twisted and deformed. The superior mesenteric artery was found at the level of the lumbar 1-2 vertebral body. Indirect portography was performed to evaluate the relationship between the portal vein and the right hepatic vein. The jugular vein was exchanged to strengthen the guide wire. After the 10F sheath was dilated, a Roups-100 sheath tube and multifunctional catheter were placed. A Roups-100 puncture needle was used to adjust the angle from the right hepatic vein. The right branch of the hepatic vein was broken into the right branch of the hepatic vein, and the guide wire catheter was used to adjust the angle. The guide wire then entered the portal vein, which was further confirmed by angiography. 


\subsection{Imaging examination}

Fourteen patients with HSOS underwent CT examination. A Philips 256-slice Brilliancei ultrafast $\mathrm{CT}$ was used for the scans after reminding the patients to start relevant scanning in breath-holding mode during quiet breathing. The patients were scanned from the diaphragm down to the lower corner of the right lobe of the liver, which was the scanning range, and 5 seconds was the average time required for these scans. The noise index value was determined to be 8.6 , the tube voltage was set to 120 $\mathrm{kV}$, the pitch was set to 1.375 , the rotation speed was set to $0.8 \mathrm{rpm} / \mathrm{s}$, the spacing was set to $5 \mathrm{~mm}$, the thickness was set to $5 \mathrm{~mm}$, and a range of 150-500 $\mathrm{mA}$ was set for milliampere automatic regulation. Dynamic contrast-enhanced scanning was performed with 920-100 $\mathrm{mL}$ of ionic contrast agent, which was injected through the median elbow vein at $2.5-3 \mathrm{~mL}$ per second. Delayed-time arterial phase scanning was performed at 20-25 seconds after the injection, portal phase scanning was performed at 55-65 seconds after injection, and delayed phase scanning was performed at 120-140 seconds after injection.

Methods: Five patients with HSOS were examined by MRI, and the relevant examinations were performed using an HDX 3.0T MR instrument developed by GE company. The following parameters were used for T1WI: TR $=180 \mathrm{~ms}$, TE was in phase, the bandwidth was 31.25 , the matrix size was $288 \times 192$, NEX was 1 , the FOV was $10 \mathrm{~cm} \times 40 \mathrm{~cm}$, the interval was $2 \mathrm{~mm}$, and the layer thickness was $8 \mathrm{~mm}$. FSPGR was used to carry out one breath-hold scan, with the following parameters: the scanning time was $16-20 \mathrm{~s}$. For T2WI, the following parameters were used: TR $=$ $4000 \mathrm{~ms}, \mathrm{TE}=85 \mathrm{~ms}$, ETL was 21, the bandwidth was 50, and the moment was $320 \mathrm{x}$ $2 \mathrm{~ms}$, the NEX was 2, the FOV was $40 \mathrm{~cm}$ x $40 \mathrm{~cm}$, and the interval was $2 \mathrm{~mm}$. The layer thickness was set to $8 \mathrm{~mm}$. Scanning was performed with FRFSE plus fat suppression for 120 to 180 seconds. The enhanced scanning conditions were TE=1.9 ms, TR $=180 \mathrm{~ms}$, matrix $=320 * 192$, bandwidth $=41.67$, NEX was set to 1 , the FOV was set to $40 \mathrm{~cm} * 40 \mathrm{~cm}$, and the interval was set to $2 \mathrm{~mm}$. The layer thickness was set to 8 mm. Scanning was performed with FRFSE plus fat suppression. A contrast agent 
(Gd-DTPA) was injected with a high-pressure syringe $(0.1 \mathrm{mmol} / \mathrm{kg})$ during enhanced scanning, and normal saline was injected at a speed of 2.0-3.0 mL per second after completion of contrast agent injection. The Gd-DTPA contrast agent was injected through the elbow vein for 20-25 seconds, then arterial phase scanning was performed (55-65 s injections were performed). A portal phase scan was performed after 55-60s, and a delayed phase scan was performed at 120-140 s after injection.

All patients were examined by two senior physicians to make a diagnosis. Enhanced and plain scan images were observed comprehensively. The density, location, size, mode and degree of enhancement of the lesions were elaborated in detail and comprehensively until a consensus was reached.

\subsection{Pathological examination}

Preoperative routine blood routine and coagulation function were examined by percutaneous puncture biopsy. Only those patients who had no coagulation dysfunction or bleeding tendency and could tolerate the operation were submitted to puncture biopsy. The patients and their families were informed about the risk of the surgery and its possible complications and signed informed consent before the operation. The following procedures were used: routine disinfection, towel laying, the placement of an ultrasonic probe coat aseptic probe cover, the installation of a special piercing frame, local anaesthesia with lidocaine, the use of a puncture biopsy needle to avoid large vessels through a normal liver parenchyma puncture site, and routine puncture by 1-2 needles. The average number of punctures was equal to the total number of punctures divided by the total number of punctures. Biopsy specimens were fixed in $10 \%$ formic acid solution and sent to the pathology department for histological examination.

\subsection{Curative effect evaluation}

Therapeutic effects were evaluated on imaging as follows. (1) Ascites after surgery was evaluated by CT examination to determine whether the ascites significantly improved or disappeared; while for ascites volume, three CT cross-sectional images 
were selected and used to measure the thickness of ascites in the bilateral subphrenic space, bilateral paracolon space and anterior bladder space (Figure 1). To estimate the volume of ascites, the standard peritoneal area in the front and back projections was assumed to be $1000 \mathrm{~cm} 2$, and the average thickness of ascites $(A+B+C+D+E) / 5$ was multiplied by this area to calculate the volume of ascites (ml) using the following formula: $(\mathrm{A}+\mathrm{B}+\mathrm{C}+\mathrm{D}+\mathrm{E}) / 5 * 1000(\mathrm{ml})$. (2) The volume of the liver was measured by CT. Compared with the preoperative volume of the liver, the postoperative volume of liver was measured. The liver volume was measured by Volume software in a CT workstation, and the threshold was set to $30-300 \mathrm{Hu}$. The axial venous phase image was delineated by the slice method. The volume of each lobe and the total liver volume were measured three times by the software, and the mean value was obtained.

(3) To evaluate liver enhancement, the liver parenchymal density and blood perfusion were observed by CT or MR enhanced scanning. For liver pathology, changes in liver microstructures between before and after the operation were evaluated.

\section{Statistical Analysis}

The statistical Department used SPSS 20.0 software to process the data. The counting data obtained before and after treatment were compared by the Kappa test, and the measurement data were tested by paired $\mathrm{T}$ tests. $\mathrm{P}<0.05$ indicates that there is a significant difference between the two groups.

\section{Results}

\subsection{CT and MR imaging analysis}

In all, data obtained in 19 patients were compared between before and after TIPS, and CT or MR showed an uneven liver density, hepatomegaly and ascites were observed before TIPS, and uneven enhancement of the liver during enhancement, a map-like liver congestion in the portal phase, and cirrhosis of the liver were observed in 8 patients. Splenomegaly was found in 4 patients with pleural effusion. Twelve cases had gallbladder wall thickening and oedema with a thickness of 5-8 mm; 6 cases had reduced oesophageal wall, gastric wall and small intestinal wall thickening and 
enhancement, which manifested as portal hypertension, and 2 cases had umbilical vein reopening. The three branches of the hepatic vein were slender, narrow or not found in 10 patients; the hepatic segment of the inferior vena cava was flat and narrow in 9 patients; the hepatic vein and inferior vena cava were patent in 14 patients, and no thrombus was found in these patients (Fig. 2A-D).

One month after TIPS, CT or MR examination showed that the liver volume of the whole group had decreased, but there was no significant difference compared with before treatment. Compared to preoperative results, postoperatively, ascites had essentially disappeared in 15 patients and was significantly decreased in 4 patients (see table 1). The enhancement of the liver parenchyma was uniform in all patients. The "map" enhancement symptoms had clearly disappeared before the operation, and the blood flow in the intrahepatic stent was smooth (Fig. 3A-C).

\subsection{Pathological changes}

Preoperative liver biopsy showed extensive oedema and degeneration, punctate or mild debris necrosis, marked congestion and expansion of the hepatic sinus, enlargement of the portal area, proliferation of fibrous connective tissue, and dilated venules in the fibrous septum (Fig. 2E-F). After TIPS treatment, liver biopsy was performed. The signs of hepatocellular oedema and degeneration were improved. Symptoms of mild congestion and dilatation were also observed in the sinuses of the liver. Compared with those observed before the operation, the dilated venules in the hepatic fibrous septum had essentially disappeared after the operation (Fig. 3D-E).

\section{Discussion}

Sinus obstruction syndrome is a rare hepatic vascular disease that may lead to complications including liver failure and portal hypertension ${ }^{[15]}$. Most of the complications observed after haematopoietic stem cell transplantation in western countries are related to the injury of sinusoidal endothelial cells by the use of a high dose of chemotherapeutic drugs before transplantation. The fatality rate of severe HSOS is as high as $80 \%{ }^{[16]}$. HSOS in developing countries mainly results in patients 
who ingested PAs, which are organic compounds synthesized by plants that are considered to be defensive compounds against herbivores ${ }^{[17]}$ and exhibit hepatotoxicity and potential carcinogenicity ${ }^{[18]}$. In China, the Chinese herbal medicine that most commonly causes HSOS and contains PAs is Tusanqi $\mathrm{i}^{[19-20]}$, which has a mortality rate of $12.2 \%-78.6 \%{ }^{[8]}$. Tusanqi, also known as chrysanthemum notoginseng, is a plant in the Sedum family. The main reason is that it is easy to use Tusanqi is that it is similar to the precious traditional Chinese medicine Panax notoginseng, which has functions including the promotion of blood circulation, the removal of blood stasis and the relief of swelling and pain. When PAs contained in herbal medicines are ingested into the body, metabolites generated under the catalysis of the hepatic cytochrome P450 enzyme combine with proteins to form pyrrole protein adducts, which damage sinusoidal endothelial cells, cause the exfoliated endothelial cells to aggregate in the central lobular area, and form cell emboli, resulting in intrahepatic congestion, liver function damage and portal hypertension ${ }^{[21-22]}$. Binding to GSH is one of the most important detoxification reactions of PA. If the level of reactive intermediates is high enough, the cell GSH pool can be depleted, leading to severe oxidative damage in liver tissue ${ }^{[23]}$. Gaohong et al. ${ }^{[24]}$ used ultra-high-performance liquid chromatography-mass spectrometry (UPLC-MS) to determine the levels of pyrrole-protein adduct in the serum of patients with HSOS induced by taking Tusanqi. It was recommended that pyrrole protein adducts could be used as a diagnostic and prognostic indicator of HSOS induced by PAs. Especially for those patients who find it difficult to recall their history of herbal preparations and food intake, this method of diagnosis can be more accurate.

The clinical manifestations of HSOS are not specific. The main manifestations of HSOS are pain in the liver area, abdominal distention, hepatomegaly, jaundice and weight gain. The clinical diagnosis of HSOS is mainly based on imaging and pathological findings. CT and MRI play an important role in the diagnosis and differential diagnosis of HSOS. Some studies have shown ${ }^{[25-26]}$ that CT is of high value in the diagnosis of HSOS. Its typical manifestations are uneven liver density, 
hepatomegaly and ascites on a plain scan, uneven liver enhancement during enhancement, and liver congestion, such as a "map-like" pattern, in the portal vein phase. Sometimes, the hepatic vein cannot be clearly visualized, and hepatic segment stenosis is observed in the inferior vena cava. The study included 11 patients who underwent upper abdominal CT examination. All patients showed uneven enhancement on a liver density test, ascites, and thinning or unclear signs in the hepatic vein, 7 had hepatomegaly, and 5 cases showed inferior vena cava stenosis. MRI was also valuable in the diagnosis of HSOS. Ye TH et al. ${ }^{[27]}$ noted that MRI was superior to CT in displaying intrahepatic veins. With the development of CT and MRI technology and the accumulation of experience by imaging doctors, liver biopsy is no longer a necessary condition for the diagnosis of HSOS. In this group, 8 cases underwent plain and enhanced upper abdominal MR imaging. Ascites and hepatic parenchyma showed uneven signals, hepatomegaly, scattered patchy low-signal shadows at the T1 stage, and cloudy high-signal shadows at the T2 stage. In 6 cases, hepatic vein thinning and inferior vena cava narrowing were observed. In imaging of HSOS patients, the hepatic vein and inferior vena cava are often not clearly visualized. It is easy to misdiagnose Budd-Chiari syndrome in the clinic. If necessary, angiographic examination can be performed to further clarify the diagnosis to avoid misdiagnosis and mistreatment. Liver puncture biopsy is a type of invasive examination. Because of the possible contraindications for liver biopsy, such as abnormal coagulation function and large amount of peritoneal effusion, this examination is often delayed, and some patients may refuse to perform liver biopsy for their own reasons. The contraindication of liver puncture was excluded for pathological examination in 19 patients in this group. Pathological findings suggest that hepatocyte oedema, hepatic sinus congestion, hepatic lobular fibrous tissue proliferation and other acute symptoms are manifestations of HSOS. Studies have shown that ${ }^{[28]}$ these manifestations can be divided into acute, subacute and chronic stages according to the pathological characteristics of HSOS, and these pathological stages have guiding significance for clinical treatment. 
There is currently no specific treatment for HSOS. Patients in the acute stage should be treated with liver protection, anticoagulation and symptomatic support to prevent liver failure. In addition to the above measures, patients in the chronic stage should receive active treatment of additional complications, such as oesophageal and gastric varices, ascites and electrolyte disorders. It is internationally believed that in patients with mild to moderate HSOS, good clinical outcomes can be obtained by removing the damaging factors, while in patients with severe HSOS, if no intervention is performed, the disease is very likely to develop into liver failure. Specifically, patients with mild HSOS can only be clinically observed. Medium-sized patients can be given small doses of diuretics to reduce the fluid load, but attention should be paid to maintaining renal blood flow to avoid pre-renal azotemia and potential hepatorenal syndrome. If ascites occurs, abdominal puncture and drainage should be performed, and the amount of ascites removed should be $1000 \mathrm{ml}$ per day. Severe HSOS must be treated with drugs or surgery. As understanding of the pathophysiological mechanism of HSOS improve, anticoagulation, thrombolysis and other therapies have gradually begun to be applied in clinical practice. However, a large number of basic and clinical studies are needed to further verify the efficacy of this treatment. It has been reported ${ }^{[8]}$ that TIPS can be used in case of poor medical treatment. It can relieve the symptoms of portal hypertension in HSOS patients and provide favourable conditions for the recovery of liver function. In 2017, a consensus on HSOS in China also noted that TIPS could be used to control intractable ascites and reduce portal hypertension in patients with HSOS caused by PAs when the efficacy of medical treatment was not good. A domestic study ${ }^{[29]}$ showed that because of the refractory ascites caused by Pas, TIPS treatment can effectively reduce the portal pressure gradient and ascites and has 1-year, 3-year and 5-year survival rates of $98 \%, 89.59 \%$ and $80 \%$, respectively. In this study, 19 HSOS patients who underwent TIPS treatment and imaging and pathological examination before and after surgery were included. No operative death occurred. A comparison of preoperative and one month post-operative imaging results showed that ascites disappeared in 15 patients and significantly decreased in 4 patients, hepatic parenchymal density or signal was more uniform after than before 
the procedure, the "map" sign and "chicken claw" sign had clearly disappeared, and the hepatic arteries and veins were naturally deformed. Pathological examination of a liver tissue puncture showed that hepatocellular oedema and hepatic sinus congestion dilatation were significantly better after than before surgery. Preoperative dilatation of small veins in the fibrous septum was seldom found. The main reason was that TIPS, as an interventional means of artificial opening the portal-hepatic vein channel, could rapidly improve the symptoms of portal hypertension and make the portal vein dilate. The venous blood flow velocity rapidly returned to normal, reduced the formation of ascites, and improved the state of liver congestion. This study effectively evaluated the efficacy of TIPS in the treatment of HSOS based on imaging and pathology.

\section{Conclusions}

Tusanqi has serious hepatotoxicity and is the main cause of HSOS in China. Traditional empirical treatment is effective in patients with mild poisoning, but severe patients often die of severe liver failure and related complications. TIPS can significantly reduce the formation of ascites and improve blood perfusion and hepatocyte oedema, thus providing favourable conditions for the recovery of liver function and improve the prognosis of patients.

\section{Abbreviations}

TIPS:Transjugular intrahepatic portosystemic shunt; HSOS:hepatic sinus obstruction syndrome; CT:Computed Tomography; MRI:Magnetic Resonance Imaging; PAs:pyrrolidine Alkaloids; DF:defibrotid; HSCT: hematopoietic stem cell transplantation; USTC:University of Science and Technology of China; Hu:hounsfield unit; GD-DTPA:Gadolinium-DTPA; FRFSE:Fast relaxation fast spin echo; NEX:Number of excitations; TR:The repetition time; TE:The ecoh time; FOV:Field of vision; ETL:Echo train length

\section{Acknowledgements}

Not applicable 


\section{Funding}

None.

\section{Consent for publication}

Not applicable.

\section{Competing interests}

The authors declare that they have no competing interests.

\section{Authors' contributions}

YuSheng He and Kaicai Liu conceived the idea for the manuscript and prepared the initial draft. Weifu Lv critically appraised the manuscript and ensured the accuracy of it's intellectual content. All authors read and approved the final manuscript.

\section{Ethics approval and consent to participate}

Acquisition of all clinical images was granted by subject verbal consent. The images in this review were obtained and accessed from the Anhui provincial hospital, The First Affiliated Hospital of USTC of Imaging local image bank. This is a repository of anonymised images accessible locally for educational purposes and no identifiable information is stored or available. Ethical approval for their use in publication was therefore deemed unnecessary. Permission for use and publication of images in the repository was granted by Weifu Lv, Head of Image center, The First Affiliated Hospital of USTC.

\section{Availability of data and materials}

All images in this review were obtained and access from Anhui provincial hospital, The First Affiliated Hospital of USTC Imaging local image bank. This is a repository of anonymised images accessible locally for educational purposes. Authorisation for their use and publication was granted by Weifu LV, Head of Image center, The First Affiliated Hospital of USTC. 


\section{References}

[1] Gao H, Na L I, Wang J Y , et al. Definitive diagnosis of hepatic sinusoidal obstruction syndrome induced by pyrrolizidine alkaloids[J]. Journal of Digestive Diseases, 2012, 13.

[2] Wang J Y, Gao H. Tusanqi and hepatic sinusoidal obstruction syndrome[J]. Journal of Digestive Diseases, 2014, 15(3):105-107.

[3]Dignan F L , Wynn R F , Hadzic N , et al. BCSH/BSBMT guideline: diagnosis and management of veno-occlusive disease (sinusoidal obstruction syndrome) following haematopoietic stem cell transplantation[J]. British Journal of Haematology, 2013, 163(4):444-457.

[4]Coppell J A , Brown S A , Perry D J . Veno-occlusive disease: cytokines, genetics, and haemostasis[J]. Blood Reviews, 2003, 17(2):63-70 .

[5] Senzolo M, Germani G, Cholongitas E, et al. Veno occlusive disease: Update on clinical management[J]. World Journal of Gastroenterology, 2007, 13(29):3918.

[6] Carreras E . Veno-occlusive disease of the liver after hemopoietic cell transplantation[J]. European Journal of Haematology, 2000, 64(5):281-291. 
[7]REN L,HU L,ZHU WD,et al.Clinical analysis of 14 cases of hepatic veno-occlusive disease[J]. Clin Focus ,2015,(1):100-103.(in Chinese)

[8]Collaborative Group of Hepatobiliary Diseases, Society of Digestive Diseases, Chinese Medical Association. Expert consensus on diagnosis and treatment of pyrrole alkaloid-related hepatic sinus obstruction syndrome (Nanjing, 2017)[J].Journal of Clinical Hepatobiliary Disease, 2017, (9): 1627-1637.(in Chinese)

[9]ZHOU HH,XU XL,GUANG L,et al.13 Cases of Hepatic Veno-occlusive Disease Caused by Radix Notoginseng and Literature Review[J]. Journal of Applied Liver Disease, 2015, 18 (3): 304-305 (in Chinese)

[10]LIN M,WU XH.Literature Analysis of 81 Cases of Hepatic Veno-occlusive Disease Caused by Tusanqi[J].Journal of Adverse Drug Reactions, 2013, 15(5):254-257. (in Chinese)

[11]Liu YL. Sinus Obstruction Syndrome: Problems and Challenges in Clinical Diagnosis and Treatment. Chinese Journal of Digestion, 2015, 35:73-76 (in Chinese)

[12] Abecasis M M, Silva J C , Ferreira I, et al. Defibrotide as salvage therapy for refractory veno-occlusive disease of the liver complicating allogeneic bone marrow transplantation[J]. Bone Marrow Transplantation, 1999, 23(8):843-846.

[13] Al B A, Al O H, Sahovic E, et al. Successful treatment of hepatic veno-occlusive disease after myeloablative allogeneic hematopoietic stem cell transplantation by early administration of a short course of methylprednisolone.[J]. Bone Marrow Transplant, 2008, 41(3):287-291. 
[14]ZHANG XT, QI XS. Recommendation of Italian Consensus on Technology, Indications and Taboos of Transjugular Intrahepatic Portosystemic Shunt 2016 [J]. Journal of Clinical Hepatobiliary Disease, 2017, 33 (3): 428-431. (in Chinese)

[15] Deleve L D , Dominique - Charles Valla, Guadalupe Garcia - Tsao. Vascular disorders of the liver[J]. Hepatology, 2009, 49.

[16]WANG XQ,QI XS,GUO XZ. Statement of the European Bone Marrow Transplantation Collaboration in 2015: Current Situation and Prospect of Hepatic Sinus Obstruction Syndrome/Hepatic Venous Obstruction [J]. Journal of Clinical Hepatobiliary Disease, 2015, (5): 659-664.(in Chinese)

[17] Macel M . Attract and deter: a dual role for pyrrolizidine alkaloids in plant-insect interactions[J]. Phytochemistry Reviews, 2011, 10(1):75-82.

[18] Rute M , David P , Valent?o Patrícia, et al. Pyrrolizidine Alkaloids: Chemistry, Pharmacology, Toxicology and Food Safety[J]. International Journal of Molecular Sciences, 2018, 19(6):1668-.

[19] Wang J Y , Gao H . Tusanqi and hepatic sinusoidal obstruction syndrome[J]. Journal of Digestive Diseases, 2014, 15(3):105-107.

[20] Wang X, Qi X , Guo X . Tusanqi-Related Sinusoidal Obstruction Syndrome in China[J]. Medicine, 2015, 94(23):e942.

[21] Yang M , Ruan J , Fu P P , et al. Cytotoxicity of pyrrolizidine alkaloid in human hepatic parenchymal and sinusoidal endothelial cells: Firm evidence for the reactive metabolites mediated pyrrolizidine alkaloid-induced hepatotoxicity[J]. Chemico-Biological Interactions, 2016, 243:119-126. 
[22]Yang M , Ruan J , Gao H , et al. First evidence of pyrrolizidine alkaloid N-oxide-induced hepatic sinusoidal obstruction syndrome in humans[J]. Archives of Toxicology, 2017.

[23] Liang Q, Sheng Y, Jiang P , et al. The gender-dependent difference of liver GSH antioxidant system in mice and its influence on isoline-induced liver injury[J]. Toxicology, 2011, 280(1-2):61-69.

[24]GAO H, WANG JY. Evidence-based clinical study of pyrrolizidine alkaloid-related sinus obstruction syndrome [J]. Journal of Gastroenterology and Hepatology, 2018, 27 (09): 979-980.(in Chinese)

[25] Kan X.Ye J , Rong X , et al.Diagnostic performance of Contrast-en hanced CT in Pyrrolizidine lkaloids-induced Hepatic Sinusoidal Obstructive Syndrome [ J ] . Sci Rep , 2016, $6: 37998$.

[26]SHI TT,HE J,SHI J, et al. CT imaging analysis of hepatic veno-occlusive disease caused by Tusanqi [J]. Journal of Practical Radiology, 2016, 32 (6): 875-878. (in Chinese)

[27]YE TH,LIANG HM,YE J, et al. CT and MRI diagnosis of hepatic sinus obstruction syndrome caused by Tusanqi. Chinese Journal of Interventional Radiology. 2015, 3 (1): 27-33.(in Chinese)

[28]SUN SZ,CHENG B. Diagnosis and differential diagnosis of hepatic vascular diseases [J].Journal of Diagnostic Pathology, 2014, 21 (6): 371-376..(in Chinese) 
[29]Luo S Chu J Huang H et al.Direct intrahepatic portocaval shunt for sinusoidal obstruction syndrome associated with hepatotoxicity of pyrrolizidine alkaloids [ J ] . Biomed Res Int , 2018, $2018: 9804582$.

\section{Figure legends}

Fig. 1 Three CT cross sections were evaluated to measure ascites thickness: the distance between the inner surface of the right abdominal wall and the surface of the liver, the distance between the inner surface of the left abdominal wall and the surface of the spleen, and the midpoint of the anterior and posterior diameters of the abdominal cavity on the horizontal plane of the left inferior renal pole. The distance between the inner surface of the right abdominal wall and the posterior edge of the right paracolon groove was measured horizontally. The distance between the vertical line $\mathrm{C}$, the distance $\mathrm{D}$ between the inner surface of the left abdominal wall and the vertical line of the posterior margin of the left paracolon sulcus, and the distance $\mathrm{E}$ between the inner surface of the anterior abdominal wall and the connecting lines of the bilateral femoral artery were measured at the horizontal level of the upper margin of the femoral head.

Fig. 2 A 62-year-old man was not able to drink Tusanqi for more than one month because of pain in both lower limbs. (A-B) The density of the liver was not uniform, and there were many ascites; the branches of the hepatic artery were enlarged and distorted in the enhanced arterial phase; the enhancement of the hepatic parenchyma in the portal vein and the delayed phase was uneven, showing patchy and map-like enhancement and periportal oedema. (C-D) The hepatic parenchyma signal and degree of enhancement around the three major hepatic veins were more uniform and clear, and the hepatic segment of the inferior vena cava was compressed and thinner. (E and F) Multiple congestion, necrosis, the local disappearance of hepatocytes and 
some fibrous proliferation in the portal area were observed in hepatic tissues; obvious expansion and congestion of the hepatic sinuses, especially around the central vein, and a small deposition of collagen fibres were found between hepatic cells (haematoxylin-eosin staining; original magnification: * 100 and $* 200$ ).

Fig.3 CT (A-C) examination of the three abovementioned patients at one month after TIPS indicated that the hepatic artery had a natural shape, the peritoneal effusion had basically disappeared, the intrahepatic density was uniform, there was no obvious abnormal density focus, and the stent blood flow was smooth. (D and E) The hepatocytes showed mild degeneration and little focal lymphocyte infiltration, some hepatic sinuses had dilated, and the arrangement of hepatocytes was slightly disordered (haematoxylin-eosin stain, original magnification: * 100 and $* 200$ ). 